\title{
Relación entre las funciones ejecutivas y el rendimiento académico en una muestra de escolares.
}

\section{Julio Flores Cuadra ${ }^{1, *}$, Marita Mojica ${ }^{1}$, Ámbar R. Perez ${ }^{2,3}$, Diana Oviedo C ${ }^{2,3}$, Gabrielle B. Britton ${ }^{3}$}

${ }^{1}$ Facultad de Psicología, Escuela de Psicología, Universidad de Panamá ${ }^{2}$ Escuela de Psicología, Facultad de Ciencias Sociales, Universidad Católica Santa María La Antigua, Panamá City, Panamá ${ }^{3}$ Centro de Neurociencias y Unidad de Investigación Clínica, INDICASAT AIP, Panamá City, Panamá.

*Autor para Correspondencia. E-mail: floresjulio27@gmail.com

Recibido: 10 de mayo de 2020

Aceptado: 23 de junio de 2020

\section{Resumen}

En la última década, la integración entre las neurociencias y la educación ha contribuido a la comprensión y mejoramiento del sistema educativo, además, se ha mostrado un interés en el estudio de las funciones ejecutivas y el rendimiento académico de los estudiantes. Las funciones ejecutivas son un sistema multimodal que coordinan procesos mentales superiores. Las investigaciones con población infantil han enfatizado la relación entre las funciones ejecutivas y las capacidades cognitivas, habilidades en lectoescritura y competencias matemáticas. Se ha reportado que alteraciones en las funciones ejecutivas podría llevar a un bajo rendimiento académico. El objetivo de este estudio fue evaluar la relación entre las funciones ejecutivas y el rendimiento académico en una muestra de escolares. Materiales y método: Se realizó un estudio descriptivo correlacional con 34 estudiantes, a los cuales se les aplicó la prueba ENFEN y se tomó el promedio de las notas del primer y segundo trimestre del año escolar como valor para medir rendimiento académico. Los datos se analizaron con el coeficiente de correlación de Spearman. Resultados: Se encontró que el desempeño de la mayoría de los estudiantes en la prueba ENFEN fue bajo y medio, mientras el rendimiento académico de los participantes fue medio y alto. No se encontraron correlaciones significativas entre la ejecución en el ENFEN y el rendimiento académico. Conclusiones: Los resultados encontrados fueron opuestos a lo planteado por la literatura, lo que conllevaría a plantear una revisión por parte del sistema educativo a la evaluación de las capacidades cognitivas y académicas de los estudiantes. Se recomienda aumentar el tamaño de la muestra y el uso de otros diseños de investigación en estudios futuros. 
Palabras clave: Funciones ejecutivas, rendimiento académico, no paramétricas, educación. Abstract

In recent years, the link between neuroscience and education has contributed to understanding and improving the educational system, moreover, an interest has been shown in the study of student's cognitive functions and academic performance. Executive functions are a multimodal system that coordinate higher mental processes. Researches have emphasized the relationship between executive function and cognitive abilities, skills competencies in literacy and mathematics. Any alteration in the executive functions cognitive domain could lead to poor academic performance. The aim of this study was to evaluate the relationship between executive functions and academic performance in a sample of elementary students. Materials and Methods: A descriptive correlational study was conducted with 34 students. Participants were assessed with the ENFEN test. In addition, their first and second trimester's grades were averaged to obtain a value for academic achievement. Data was analyzed with the Spearman correlation coefficient. Results: The performance of most students in the ENFEN test was low and medium. Academic performance was medium and high. No significant correlations between ENFEN scores and averaged grades were found. Conclusions: The results found were the opposite of those proposed by the literature, which could suggest a review of the evaluation of students' cognitive and academic abilities by the educational system. We recommend increasing the sample size and the use of other research designs in future studies.

Keywords: executive functions, academic performance, non-parametric, education.

\section{Introducción}

A medida que el cerebro se desarrolla durante la gestación y la infancia, se evidencia una mayor sinaptogénesis y mielinización (Sánchez-Carpintero \& Narbona, 2004) en distintas áreas cerebrales. Estas regiones se desarrollan a diferentes velocidades, siendo la corteza prefrontal una de las últimas áreas cerebrales en madurar. La formación y organización de las sinapsis en el área prefrontal se asocia al desarrollo de las funciones ejecutivas (Lázaro \& Ostrosky-Solís, 2012; Portellano et al., 2009). Las funciones ejecutivas son un sistema multimodal que coordinan procesos mentales superiores, permiten resolver problemas complejos, inhibir patrones inadecuados de conducta, tomar decisiones y alternar entre diferentes tipos de información (Ardila \& Ostrosky-Solís, 2012). Por lo tanto, estas se relacionan y coordinan otros procesos cognitivos como la memoria de trabajo, atención selectiva y sostenida, flexibilidad mental, inhibición y planificación (Portellano et al., 2009).

Estudios sugieren que aproximadamente a los dos años y medio, la memoria de trabajo adquiere mayor capacidad, lo cual permite que la información relevante se mantenga más tiempo en el componente de memoria para realizar una acción (Rosselli, M., Matute, E., Ardila, A., 2010). Por otro lado, entre los seis y ocho años, la capacidad para inhibir patrones de conducta inadecuados aumenta y a los doce años alcanza su máximo desarrollo. Además, el proceso de planificación se vuelve más eficiente y enfocado a objetivos (Rosselli, M., Matute, E., Ardila, A., 2010). En el ámbito educativo esto permite que los niños desarrollen su capacidad de regular, programar, tomar decisiones y verificar sus pensamientos y conducta en el proceso de enseñanza-aprendizaje (Herreras, 2014).

En las últimas dos décadas, las neurociencias han trabajado en la comprensión y mejora de la educación (Santiago-Ramajo \& Martín-Lobo, 2018). Actualmente se resalta la necesidad que el estudiante disponga de un adecuado nivel de maduración neuropsicológica para hacerle frente al proceso de enseñanza-aprendizaje (Portellano, J. et. al. 2008). Diversas investigaciones se han centrado en el estudio de las funciones ejecutivas y su relación con el rendimiento académico, especialmente en 
asignaturas como matemáticas, español, inglés y ciencias naturales (Castillo-Parra, Gómez-Pérez, \& Ostrosky-Solís, 2009; Obredor, C. 2015; Fonseca, Rodríguez, \& Parra, 2016). Estos estudios han señalado que cualquier alteración que afecte los procesos que coordinan las funciones ejecutivas, podría asociarse al bajo rendimiento académico de un estudiante (Fonseca et al., 2016). Es esencial que los profesionales que se desempeñan en centros educativos conozcan que el proceso de enseñanza-aprendizaje está influenciado por factores biológicos, psicológicos y sociales (Portellano et al., 2009).

La Dirección de Planificación del Ministerio de Educación de Panamá, reportó 16,023 estudiantes reprobados en el año 2016, 17,089 en el 2017 y 16,689 en el 2018 a nivel de primaria (MEDUCA, 2018). Las materias en las que se reporta mayor dificultad son matemáticas y español (Barría, 2016). En Panamá, las investigaciones sobre las posibles causas del bajo rendimiento académico se han enfocado en los hábitos de estudio, niveles de ansiedad, estructura familiar y motivación (Corro, 2016; Lashington, 2016; Otero, 2014). Sin embargo, las investigaciones con enfoque neuropsicológico en el área educativa son limitadas, por lo tanto, el objetivo principal de este estudio fue evaluar la relación entre las funciones ejecutivas y el rendimiento escolar por materias en una muestra de escolares de un colegio público de la ciudad de Panamá.

\section{Materiales y método}

\section{Diseño de investigación}

Se realizó un estudio descriptivo correlacional con diseño no experimental transversal.

\section{Muestra}

El estudio tuvo una muestra de 34 estudiantes de un colegio oficial de la ciudad de Panamá, entre las edades de 9 a 12 años, con una media de edad de 10.53 (DE=1.02). El 58.8\% eran mujeres y 41.2\% eran hombres. La muestra se distribuyó de la siguiente manera: 9 años (17.6\%), 10 años (32.4\%), 11 años $(29.4 \%)$ y 12 años $(20.6 \%)$.

El muestreo fue por conveniencia. Los criterios de inclusión fueron que los padres de los estudiantes firmaran un consentimiento informado, y que los participantes participaran voluntariamente y no presentaran limitaciones físicas, cognitivas, perceptivas, visuales, auditivas o motoras que limitaran la ejecución de la prueba.

\section{Instrumentos}

Se utilizó la prueba Evaluación Neuropsicológica de las Funciones Ejecutivas en Niños (ENFEN), compuesta por 4 subpruebas que permiten evaluar funciones ejecutivas (FFEE) en estudiantes de habla hispana de 6 a 12 años (Portellano et al., 2009). La aplicación fue individual con una duración entre 20 y 30 minutos. Las subpruebas utilizadas incluyen: fluidez fonológica (F1), fluidez semántica (F2), senderos grises (S1), senderos a color (S2), anillas (A) e interferencia (I).

En la subprueba de fluidez (F1), el estudiante debía decir todas las palabras posibles que iniciaran con la letra " $m$ " durante un minuto. En fluidez semántica (F2), el estudiante tenía que decir todos los animales que se le ocurrieran en un periodo de un minuto, sin importar que tipo de animal fuera y por cual letra iniciaba. En la subprueba de senderos grises (S1), el estudiante tenía que unir con líneas siguiendo el orden de los números del 20 al 1 (mayor a menor), lo más rápido que pudiera. En la 
subprueba senderos a color (S2), el estudiante debía unir con líneas del 1 al 21, pero intercalando entre los colores rosado - amarillo y siguiendo el orden de los números (menor a mayor). En la subprueba de anillas (A) el estudiante ordenaba una serie de anillas de diferentes colores (amarilla, roja, azul, blanca, negra y verde) lo más rápido que podía, según los modelos que le mostraba el evaluador, pasándolas de una columna a otra sobre un bloque de tres columnas. El evaluador colocaba las anillas en la columna del lado izquierdo del estudiante y este las movía a la columna del lado derecho, ubicando las anillas igual al modelo que el evaluador mostró. La subprueba de interferencia (I), sigue una ejecución similar a la prueba de Stroop, donde el escolar tenía que decir lo más rápido que pudiera el color en el cual la palabra estuviera escrita en vez de nombrar la palabra, en una lista de tres columnas que debía ser leída de arriba hacia abajo.

Por otro lado, para el registro de calificaciones se tomó el promedio de las notas del periodo académico del primer y segundo trimestre del 2018, para matemáticas, español, inglés y ciencias naturales (MEDUCA, 2018).

\section{Análisis estadístico}

Se hizo un análisis descriptivo de las variables, donde se mostró el desempeño de la prueba ENFEN según la edad en medianas. Luego, cada una de las puntuaciones obtenidas en la prueba ENFEN y el rendimiento académico, se distribuyó en rangos (tabla 1) para ser presentados en porcentajes. Posteriormente, se realizó una prueba de normalidad con la prueba Kolmogorov-Smirnov, que evidenció una muestra no paramétrica por lo que se utilizó el estadístico Rho de Spearman para conocer las asociaciones entre las variables.

\section{Tabla 1}

Rangos establecidos para la medición de las variables

\begin{tabular}{|c|c|c|}
\hline \multicolumn{3}{|c|}{ Variables de investigación } \\
\hline \multicolumn{2}{|c|}{$\overrightarrow{\text { Funciones ejecutivas }}$} & Alto: Obtiene una valoración numérica de 10, 9, 8 o 7 en \\
\hline Prueba & Evaluación & Medio: Obtiene una valoración numérica de 6,5 o 4 en \\
\hline Neuropsicológica & de & los indicadores de desempeño de la prueba ENFEN. \\
\hline $\begin{array}{l}\text { Funciones } \\
(\text { ENFEN) }\end{array}$ & Ejecutivas & $\begin{array}{l}\text { Bajo: Obtiene una valoración numérica de 3, } 2 \text { o } 1 \text { en los } \\
\text { indicadores de desempeño de la prueba ENFEN. }\end{array}$ \\
\hline
\end{tabular}

\section{Rendimiento académico}

Boletín de Calificaciones: Registro de Calificaciones.
Alto: Obtiene una valoración numérica de 4.1 - 5.0 en los indicadores de desempeño en el proceso de aprendizaje en las materias.

Medio: Obtiene una valoración numérica de 3.0 - 4.0 en los indicadores de desempeño en el proceso de aprendizaje en las materias.

Bajo: Obtiene una valoración numérica de 1.0 - 2.9 en los indicadores de desempeño en el proceso de aprendizaje de las materias.

Fuente: Elaboración Propia. Adaptado de Portellano, Martínez y Zumárraga (2009). Y el registro de calificaciones de los estudiantes 


\section{Resultados}

En la tabla 2, se muestra el desempeño de la prueba ENFEN en cada una de las subescalas según la edad de los participantes. Los estudiantes de 9 años se desempeñaron mejor que los estudiantes de otras edades en las subescalas de fluidez fonológica (4.50), senderos a color (5.00), anillas (4.50). Por otro lado, en las subescalas fluidez semántica (3.00-4.00) e interferencia $(3.00-4.00)$ se observa un desempeño similar en todas las edades. Por último, en senderos grises (6.00), los estudiantes de 12 años se desempeñaron mejor que los de las otras edades.

\section{Tabla 2}

Desempeño en la prueba ENFEN en medianas según edad de los participantes

\begin{tabular}{|c|c|c|c|c|c|c|}
\hline \multirow{3}{*}{ Edad } & \multicolumn{6}{|c|}{ Desempeño Prueba ENFEN } \\
\hline & \multicolumn{6}{|l|}{ F1 } \\
\hline & & F2 & S1 & $\mathbf{S} 2$ & $\mathbf{A}$ & I \\
\hline 9 años $(\mathrm{n}=6)$ & 4.50 & 4.00 & 4.00 & 5.00 & 4.50 & 4.00 \\
\hline 10 años & & & & & & \\
\hline$(n=11)$ & 3.00 & 4.00 & 5.00 & 3.00 & 2.00 & 3.00 \\
\hline 11 años & & & & & & \\
\hline $\begin{array}{l}(\mathrm{n}=10) \\
12\end{array}$ & 3.00 & 3.00 & 3.50 & 2.00 & 3.50 & 3.00 \\
\hline$(n=7)$ & 3.00 & 4.00 & 6.00 & 4.00 & 3.00 & 4.00 \\
\hline
\end{tabular}

Resultados en medianas de la prueba ENFEN en cada una de las subescalas, según la edad de los estudiantes. Desempeño alto (10,9,8 o 7), desempeño medio (6, 5 o 4), desempeño bajo (3, 2 o 1). F1= fluidez fonológica, F2= fluidez semántica, $S 1=$ senderos grises, $S 2=$ senderos a color, $A=$ anillas, $I=$ interferencia.

En la figura 1, se observa que la mayoría de la muestra se desempeñó en los rangos medio ( 40\%) y bajo ( 50\%). En fluidez fonológica (F1), el 67.60\% obtuvo un desempeño bajo. En fluidez semántica (F2) el 47.10\% obtuvo un desempeño medio, en contraste con un $44.10 \%$ que fue bajo. En la subprueba de senderos gris (S1), el $38.20 \%$ obtuvo un desempeño medio y un $35.30 \%$ un desempeño bajo. Por otro lado, en senderos a color (S2), 55.90\% obtuvo un desempeño bajo. En la subprueba de anillas (A), el 50\% obtuvo un desempeño medio y el otro 50\% fue bajo. Por último, en la subprueba de interferencia (I) el 52.90\%, obtuvo un desempeño bajo 


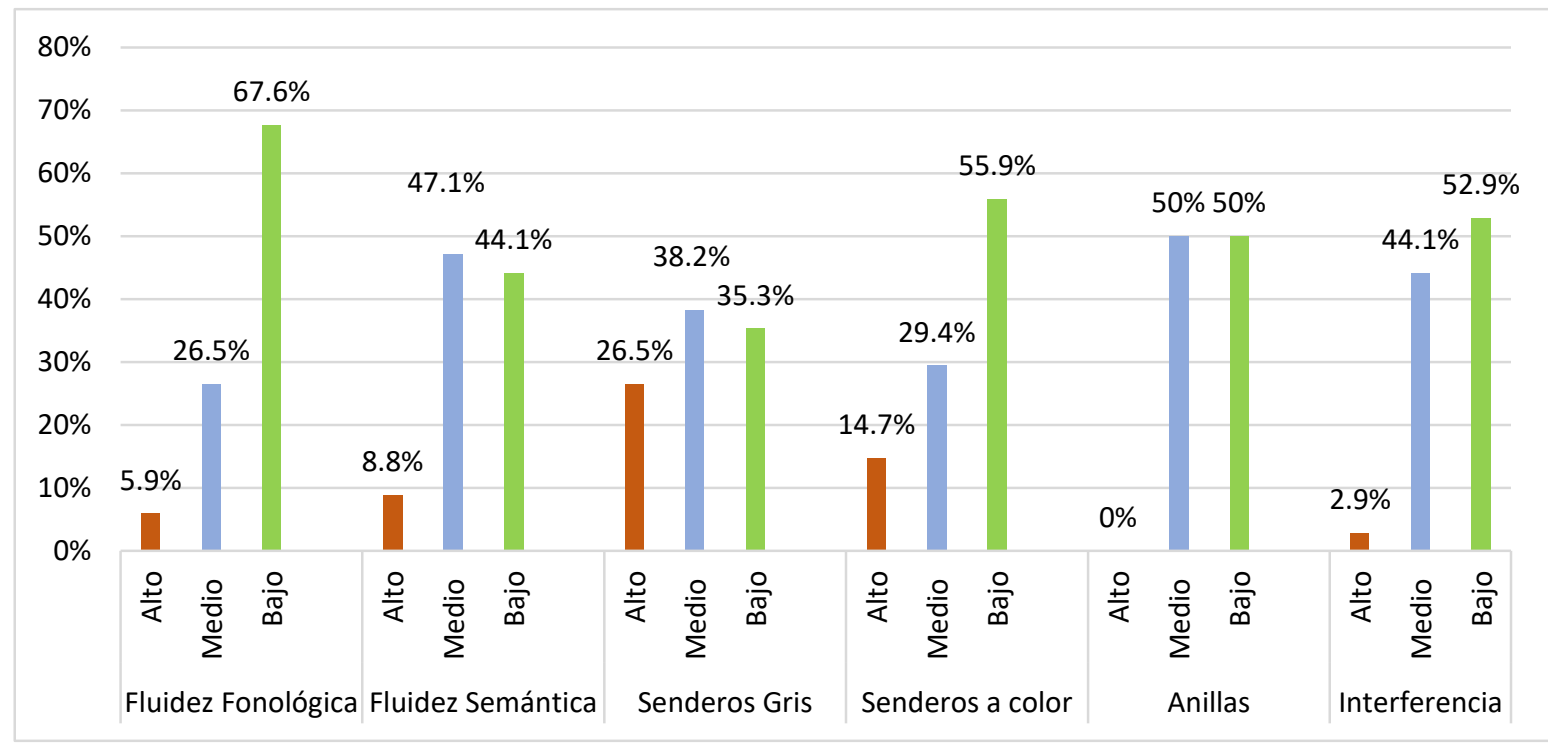

Figura 1: Desempeño de la muestra en cada subprueba de la prueba ENFEN.

En la figura 2, se muestra el rendimiento académico de los participantes por materias (español, matemáticas, inglés y ciencias naturales). El 52.94\% obtuvo un desempeño alto en la materia de español. En matemáticas, el 41.18\% obtuvo un desempeño alto y el 50\% un desempeño medio.

En inglés, el $47.06 \%$, obtuvo un desempeño alto y el 52.94\% un desempeño medio. Por último, el $64.71 \%$ obtuvo un desempeño alto en ciencias naturales.

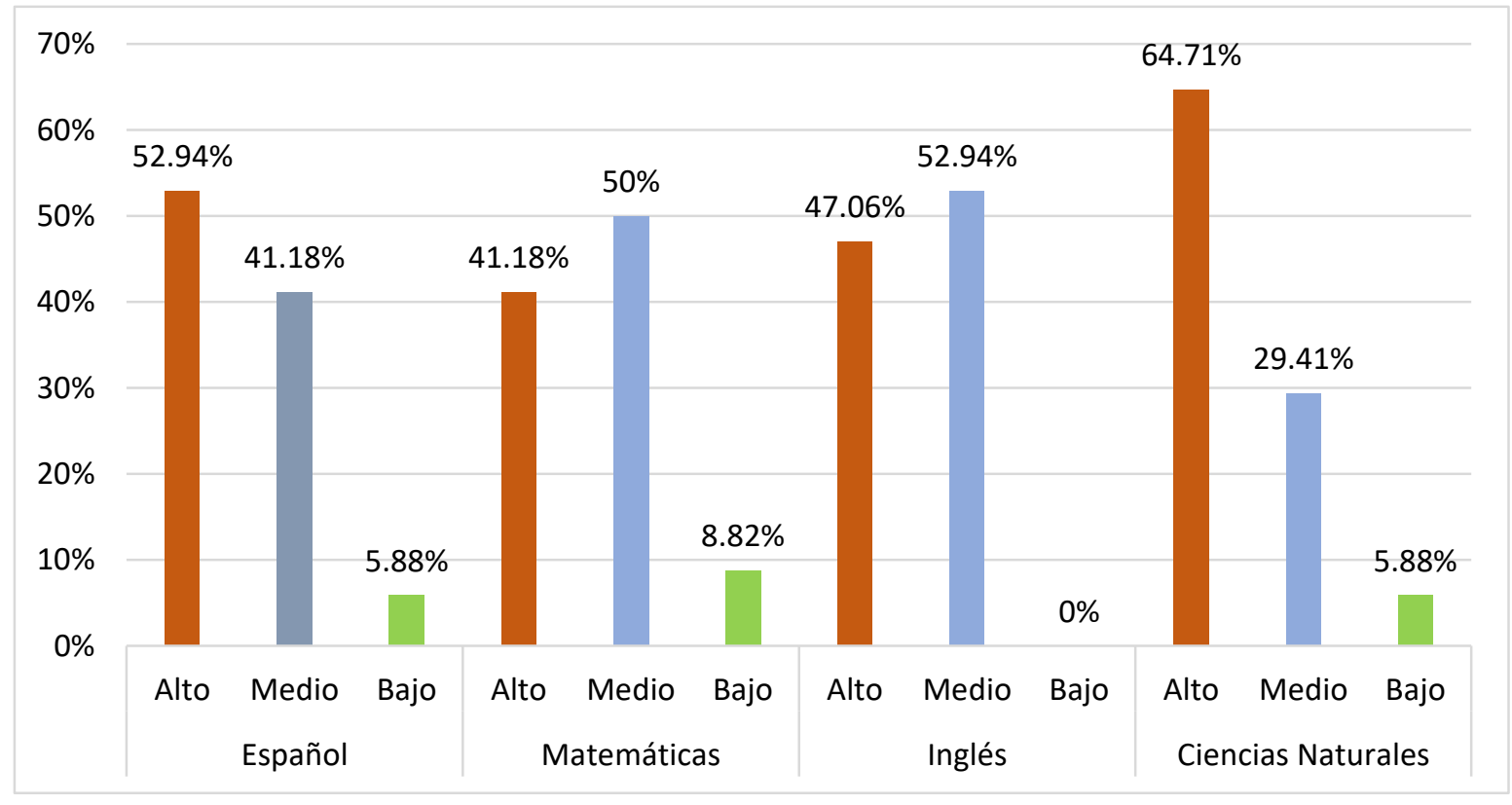

Figura 2: Rendimiento académico de la muestra del primer trimestre y segundo trimestre del año 2018 de los estudiantes de una escuela oficial de la República de Panamá, Ciudad de Panamá. 
En la tabla 3, se puede observar que no se encontraron correlaciones significativas entre las funciones ejecutivas y el rendimiento académico de los estudiantes ( $\mathrm{p}>0.05)$. No existió asociación estadística entre las variables de investigación.

Tabla 3:

Matriz. de correlaciones entre las funciones ejecutivas y el rendimiento académico en una muestra de escolares

\begin{tabular}{llllll}
\hline \multirow{2}{*}{ Subpruebas } & Rho Spearman & Español & Matemáticas & Inglés & $\begin{array}{l}\text { Ciencias } \\
\text { Naturales }\end{array}$ \\
\hline \multirow{2}{*}{ Fluidez Fonológica } & Rho & 0.307 & 0.159 & 0.022 & 0.157 \\
& $p$ & 0.078 & 0.37 & 0.902 & 0.374 \\
\hline \multirow{2}{*}{ Fluidez Semántica } & $R h o$ & -0.161 & 0.049 & -0.06 & -0.094 \\
& $p$ & 0.362 & 0.782 & 0.736 & 0.597 \\
\hline \multirow{2}{*}{ Senderos Gris } & Rho & 0 & 0.09 & 0.096 & 0.068 \\
& $p$ & 1 & 0.613 & 0.589 & 0.703 \\
\hline \multirow{2}{*}{ Senderos color } & Rho & -0.158 & 0.118 & 0.222 & -0.028 \\
& $p$ & 0.373 & 0.507 & 0.207 & 0.874 \\
\hline \multirow{2}{*}{ Anillas } & Rho & -0.108 & -0.07 & 0 & -0.043 \\
\multirow{2}{*}{ Interferencia } & $p$ & 0.541 & 0.693 & 1 & 0.81 \\
\hline Rho: Coefciente & Rho & 0.21 & 0.154 & 0.254 & 0.125 \\
\hline
\end{tabular}

Rho: Coeficiente de correlación

p: Significancia estadística $(p<0.05)$

\section{Discusión}

El objetivo de este estudio fue evaluar la relación entre las funciones ejecutivas y el rendimiento escolar por materias en una muestra de estudiantes de un colegio público de la ciudad de Panamá. Se planteó que existiría relación entre las funciones ejecutivas y rendimiento académico y que los estudiantes que tuvieran mejor rendimiento académico tendrían un mejor resultado en la prueba ENFEN (correlación positiva). Sin embargo, los resultados señalan que no hubo relación entre las funciones ejecutivas y el rendimiento escolar de la muestra.

En cuanto a los resultados obtenidos en la prueba ENFEN (tabla 2), los estudiantes de 9 años obtuvieron mejores puntuaciones en comparación con las otras edades. Este resultado no va acorde con lo expuesto por teorías que resaltan que la maduración de la corteza prefrontal y las conexiones sinápticas se potencian mientras se avanza de edad, mejorando no solamente las funciones ejecutivas, sino el funcionamiento cognoscitivo en general con el aumento de la edad (Freund et al., 2010; Gooch et al., 2016; Nakamura et al., 2001; Rosselli et al., 2008). También, se esperaba que los estudiantes de mayor edad, obtuvieran un mayor desempeño en la prueba ENFEN, como es reportado en otros estudios (Fonseca et al., 2016). No obstante, solo en la prueba de senderos grises, la cual evalúa flexibilidad cognitiva y toma de decisiones (Portellano et al., 2009) los estudiante de 12 años se desempeñaron mejor que las otras edad. Por otro lado, el desempeño académico en la mayoría de los estudiantes fue entre medio y alto (figura2), en contraste con el desempeño en las funciones ejecutivas, el cual fue entre bajo y medio (figura 1). Esto no va de acorde a lo evidenciado en otros estudios, donde se ha observado que mientras se avanza de grado educativo, las funciones ejecutivas son de 
importancia para el éxito académico (Castillo-Parra et al., 2009). Se ha evidenciado que a los 6 años, la memoria de trabajo está relacionada con la capacidad de lectura, escritura y matemáticas (Fonseca et al., 2016; Lan et al., 2011), y a partir de los 10 años, la flexibilidad mental, planificación e inhibición son de relevancia para el proceso de enseñanza-aprendizaje (Fonseca et al., 2016; Hooper et al., 2002; St Clair-Thompson \& Gathercole, 2006).

Sin embargo, a pesar de que la mayoría de los estudios reportados han encontrado relación entre las funciones ejecutivas y el rendimiento académico, existen investigaciones con resultados similares a los obtenidos en esta investigación. Por ejemplo, se ha reportado que las funciones ejecutivas no inciden como variables predictoras en la estimación del desempeño académico en las asignaturas matemáticas y lenguaje en una muestra de estudiantes de tercer grado (Obredor, C. 2015). En otro estudio tampoco se encontró relación entre las funciones ejecutivas y el desempeño escolar (Merchán, 2016). Además, se observó que en cuanto al funcionamiento ejecutivo, tomando en cuenta el sexo de los participantes no existen diferencias, al igual que con otras investigaciones (Barceló, Lewis, \& Moreno, 2006; Vergara, M.I., 2011; Franco, F.C. 2016).

Existen distintas posibles explicaciones para estos resultados. Primeramente, en un estudio reciente, se ha evidenciado que el desempeño en las funciones ejecutivas solo explica 15\% del rendimiento escolar del estudiante (Santiago-Ramajo \& Martín-Lobo, 2018), lo que sugiere que existen otras variables personales, sociofamiliares, psicológicas y educativas que contribuyen al rendimiento escolar del estudiante (Diamond, 2013; Portellano et al., 2009; Rueda, 2014). Segundo, se ha propuesto que la actividad escolar, es decir, la estimulación que recibe el estudiante en el salón de clases para que sea parte del proceso de enseñanza-aprendizaje, tiene un papel más prioritario que el nivel educativo del estudiante, lo cual produce diferencias en el funcionamiento ejecutivo en jóvenes de diferentes niveles académicos (Flores et al., 2011). Por lo tanto, es necesario que se haga una evaluación al sistema educativo panameño sobre los métodos en los cuales se evalúa el rendimiento académico y, por otro lado, enfocarse en potenciar las habilidades cognitivas de los estudiantes.

Las limitaciones de este estudio son varias. Por un lado, el tamaño de la muestra fue reducido, por lo que resultados no pueden generalizarse al resto de la población. La evaluación fue en un único momento. La aplicación de la prueba ENFEN fue solamente en un colegio oficial y no se tomó en cuenta todo el rango de edades que abarca la formación de primaria, media y premedia.

Una de las principales fortalezas de este estudio es el aporte teórico a futuras investigaciones relacionadas al estudio de variables neuropsicológicas en el ámbito educativo, ya que en Panamá estos estudios son escasos. La adquisición de pruebas neuropsicológicas de los gabinetes psicopedagógicos es esencial para evaluar las capacidades cognitivas de los estudiantes y fomentar la investigación neuropsicológica en las escuelas del país (Santos, 2015). Se recomienda continuar esta línea de investigación, aumentando el tamaño de la muestra e incluyendo estudiantes de escuelas públicas y privadas para realizar diferentes diseños de investigación y la utilización de una batería de pruebas neuropsicológicas.

\section{Agradecimientos}

Se agradece a todo el plantel educativo que colaboraron con la realización de esta investigación, especialmente a la Lic. Diana González por su orientación para conseguir los permisos por parte del MEDUCA para la realización de este estudio. Los autores agradecen también el apoyo financiero del Sistema Nacional de Investigación (SNI), de la Secretaría Nacional de Ciencia, Tecnología e 
Innovación (SENACYT). A la Universidad Santa María La Antigua (USMA) y al Instituto de Investigaciones Científicas y Servicios de Alta Tecnología (INDICASAT).

\section{Referencias}

Ardila, A., \& Ostrosky-Solís, F. (2012). Guía para el diagnóstico neuropsicológico. Miami, Florida, EE.UU. \& México, D.F.

Barceló, E., Lewis, S., \& Moreno, M. (2006). Funciones ejecutivas en estudiantes universitarios que presentan bajo y alto rendimiento académico. Psicología Desde El Caribe.

Barría, G. L. (16 de diciembre de 2016). Aumentan fracasos escolares. La estrella de Panamá.

Castillo-parra, G., Gómez-Pérez, E., \& Ostrosky-Solís, F. (2009). Relación entre las funciones cognitivas y el nivel de rendmiento académico en niños. Revista Neuropsicología, Neuropsiquiatría y Neurociencias. Abril 2009, Vol.9, No1, pp. 41-54

Cirly Paola Obredor Ávila. (2015). Papel Predictor de las Funciones Ejecutivas en el Desempeño Académico en las áreas de Matemática y Lenguaje en estudiantes de Tercer Grado de Educación Básica Primaria. Universidad de la Costa - Barranquilla.

Corro, N. (2016). Estudio descriptivo sobre los niveles de ansiedad en una muestra de niños de sexto grado y como se relaciona con la estructura familiar. Tesis de grado. Panamá

Diamond, A. (2013). Executive Funtions. Annual Reviews Psychology, 64, 135-168. https://doi.org/10.1146/annurev-psych-113011-143750

Flores, J., Tinajero, B., \& Castro, B. (2011). Influencia del Nivel y de la Actividad Escolar en las Funciones Ejecutivas. Interamerican Journal of Psychology, 45(2), 281-292.

Fonseca, G. P., Rodríguez, L. C., \& Parra, J. H. (2016). Relación Entre Funciones Ejecutivas Y Rendimiento Académico Por Asignaturas En Escolares De 6 a 12 Años. Hacia La Promoción de La Salud, 21(2), 41-58. https:/ / doi.org/10.17151/hpsal.2016.21.2.4

Franco, F. C. (2016). Funciones ejecutivas y desempeño academico en estudiantes de primer año de psicologia de la corporacion universitaria minuto de dios, en bello antioquia. Universidad Internacional de la Rioja.

Freund, L. S., Bierman, K. L., \& Torres, M. (2010). Promoting the Development of Executive Functions through Early Education and Prevention Programs Karen L. Bierman \& Marcela Torres, 1-41.

Gooch, D., Thompson, P., Nash, H. M., Snowling, M. J., \& Hulme, C. (2016). The development of executive function and language skills in the early school years. Journal of Child Psychology and Psychiatry and Allied Disciplines, 57(2), 180-187. https://doi.org/10.1111/jcpp.12458

Hernández, R., Fernández, C., Baptista, M. (2014). Metodología de la Investigación. Sexta Edición. México: McGraw Hill.

Hooper, S. R., Swartz, C. W., Wakely, M. B., De Kruif, R. E. L., \& Montgomery, J. W. (2002). Executive functions in elementary school children with and without problems in written expression. Journal of Learning Disabilities, 35(1), 57-68. 
https://doi.org/10.1177/002221940203500105

Lan, X., Legare, C. H., Ponitz, C. C., Li, S., \& Morrison, F. J. (2011). Investigating the links between the subcomponents of executive function and academic achievement: A cross-cultural analysis of Chinese and American preschoolers. Joumal of Experimental Child Psychology, 108(3), 677-692. https://doi.org/10.1016/j.jecp.2010.11.001

Lashington, E. (2016). La autoestima y el rendimiento académico en una muestra de estudiantes de séptimo año de un colegio público de la ciudad de Panamá. Tesis de grado . Panamá

Lázaro, J. C. F., \& Ostrosky-Solís, F. (2012). Desarrollo neuropsicológico de las lóbulos frontales y funciones ejecutivas (Primera Ed). México: Manual Moderno.

MEDUCA. (2018). Cantidad de Estudiantes Aprobados, Reprobados, Desertores en la Educación Primaria en la República de Panamá. Panamá: Dirección de Planificación. Departamento de estadística.

Merchán, N. G. (2016). Funciones ejecutivas y desempeño academico en estudiantes de primer año de psicologia de la corporacion universitaria minuto de dios, en bello antioquia. Universidad Internacional de la Rioja.

Obredor, C. (2015). Papel predictor de las funciones ejecutivas en el desempeño académico en las áreas de matemática y lenguaje en estudiantes de tercer grado de educación básica primaria. Universidad de la Costa. Barranquilla, Colombia.

Otero, Y. (2014). Estudio correlacional entre las variables autoconcepto, motivación y autorregulación con el rendimiento académico en estudiantes. Tesis de grado. Panamá

Portellano, J. A., Martinez, A., \& Zumárraga, A. (2009). Evaluación de las Funciones Ejecutivas en niños. 1 ed. Madrid, España: TEA Ediciones

Rosselli, M., Jurado, M. B., \& Matute, E. (2008). Las Funciones Ejecutivas a través de la Vida. Revista Neuropsicología, Neuropsiquiatría y Neurociencias, 8(1), 23-46. Retrieved from http://neurociencias.udea.edu.co/revista/PDF/REVNEURO vol8 num1 5.pdf

Rosselli, M., Matute, E., Ardila, A. (2010). Neuropsicología del desarrollo infantil. México D.F.: El Manual Moderno S.A.de C.V.

Rueda, P. O. (2014). Competencias básicas y procesos perceptivos: Factores claves en la formación y orientación de los jóvenes en riesgo de exclusión educativa y sociolaboral. Revista de Investigacion Educativa, 32(2), 531-546. https://doi.org/10.6018/rie.32.2.181551

Sánchez-Carpintero, R., \& Narbona, J. (2004). El sistema ejecutivo y las lesiones frontales en el niño. Revista de Neurologia, 39(2), 188-191.

Santiago-Ramajo, S., \& Martín-Lobo, M. P. (2018). Relation between Executive Functions and Empathy and Their Influence on Academic Performance in Students of Basic Vocational Training. Electronic Journal of Research in Educational Psychology, 16(46), 517-536.

Santos, I. A. R. (2015). La evaluación neuropsicológica infantil en México. Ciencia y Futuro, 5(2), 96111.

St Clair-Thompson, H. L., \& Gathercole, S. E. (2006). Executive functions and achievements in school: Shifting, updating, inhibition, and working memory. Quarterly Journal of Experimental 
Psychology, 59(4), 745-759. https://doi.org/10.1080/17470210500162854

Vergara, M. I. (2011). funciones ejecutivas y desempeño academico en estudiantes de primer año de psicologia de la corporacion universitaria minuto de dios, en bello antioquia. Medellín, Colombia: Universidad de San Buenaventura. 